\title{
New Phase Fitted and Amplification Fitted Numerov-Type Methods for Periodic IVPs with Two Frequencies
}

\author{
Yonglei Fang, ${ }^{1}$ Xiong You, ${ }^{2}$ and Zhaoxia Chen $^{2}$ \\ ${ }^{1}$ School of Mathematics and Statistics, Zaozhuang University, Zaozhuang 277160, China \\ ${ }^{2}$ Department of Applied Mathematics, Nanjing Agricultural University, Nanjing 210095, China
}

Correspondence should be addressed to Xiong You, youx@njau.edu.cn

Received 8 April 2012; Revised 20 June 2012; Accepted 28 August 2012

Academic Editor: Josip E. Pecaric

Copyright (C) 2012 Yonglei Fang et al. This is an open access article distributed under the Creative Commons Attribution License, which permits unrestricted use, distribution, and reproduction in any medium, provided the original work is properly cited.

Phase fitted and amplification fitted Numerov-type methods for periodic initial value problems with two frequencies are investigated. A one-frequency method and a two-frequency method are constructed. The two new methods both have algebraic order five and are dispersive of order six and dissipative of order five. The two-dimensional absolute stability region for the one-frequency method and the three-dimensional absolute stability region for the two-frequency method are plotted. Numerical experiments are reported to show the efficiency and competence of the two new methods.

\section{Introduction}

In this paper we are interested in the numerical integration of the initial value problem (IVP) of ordinary differential equations in the form

$$
y^{\prime \prime}(x)=f(x, y), \quad y\left(x_{0}\right)=y_{0}, \quad y^{\prime}\left(x_{0}\right)=y_{0}^{\prime}, \quad x \in\left[x_{0}, x_{\text {end }}\right],
$$

whose solutions exhibit a pronounced oscillatory character. Such problems are frequently encountered in a variety of scientific fields and engineering applications. Since the analytical solutions of these equations are usually not available, the numerical solutions become very important. To our knowledge, most existing methods of fitted type (trigonometrically/exponentially fitted, phase fitted and amplification fitted, etc.) are adapted to one frequency (see [1-3]). We call them one-frequency methods. For example, by phase fitting, Van de Vyver [4] constructed an adapted explicit Numerov-type method for oscillatory 
problems with one frequency. Other related work can be found in [5-20]. However, for oscillatory problems with many frequencies in the solution, when we apply a one-frequency method, it is difficult to choose the most suitable fitting frequency. Contrast to the onefrequency methods, papers on integrators that are adapted to more than one frequency are few. In [21], Wang obtained a trigonometrically fitted Numerov-type method for periodic IVPs with two frequencies and provided some numerical examples to show that his method associated with two frequencies is more accurate and more efficient than the trigonometrically fitted Numerov methods with one frequency. More recently, Fang et al. [22] gave a trigonometrically fitted explicit Numerov-type method for the numerical integration of oscillatory problems with two frequencies. Inspired by Van de Vyver's idea in [4], we investigate, in this paper, explicit Numerov-type methods for the numerical integration of periodic IVPs with two frequencies. The paper is organized as follows. In Section 2, the preliminaries of stability and phase properties of explicit Numerov-type methods are recalled. In Section 3, we construct two new phase fitted and amplification fitted explicit Numerov-type methods for periodic initial value problems with two frequencies. In Section 4, the stability and phase properties of the newly derived methods are analyzed. In Section 5, we present some numerical results. Section 6 is devoted to the conclusions.

\section{Numerov-Type Methods}

In this section, we recall some elementary notions about Numerov-type methods. The explicit Numerov-type methods considered here have the form

$$
\begin{gathered}
Y_{i}=\left(1+c_{i}\right) y_{n}-c_{i} y_{n-1}+h^{2} \sum_{j=1}^{s-1} a_{i j} f\left(x_{n}+c_{j} h, Y_{j}\right), \quad i=1, \ldots, s . \\
y_{n+1}=2 y_{n}-y_{n-1}+h^{2} \sum_{i=1}^{s} b_{i} f\left(x_{n}+c_{i} h, Y_{i}\right),
\end{gathered}
$$

with $c_{1}=-1, c_{2}=0$, and can be expressed in the Butcher tableau as

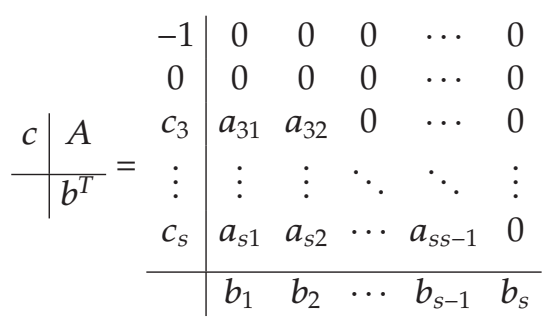

or equivalently by the triplet $(c, A, b)$. The order conditions for this kind of Numerov-type methods can be found in [23]. A favorable property of these methods is that they need only $s-1$ function evaluations at each step. According to the idea of Lambert and Watson [24], the stability of the Numerov-type methods can be analyzed by the test equation

$$
y^{\prime \prime}(x)=-\lambda^{2} y(x)
$$


Applying the explicit Numerov-type method (2.1) to (2.3), we obtain the following relation

$$
y_{n+1}-S\left(H^{2}\right) y_{n}+P\left(H^{2}\right) y_{n-1}=0, \quad H=\lambda h
$$

with

$$
S\left(H^{2}\right)=2-H^{2} b^{T}\left(I+H^{2} A\right)^{-1}(e+c), \quad P\left(H^{2}\right)=1-H^{2} b^{T}\left(I+H^{2} A\right)^{-1} c
$$

Since the coefficient matrix $A$ satisfies $A^{s-1}=0$, we have

$$
\begin{gathered}
S\left(H^{2}\right)=2-H^{2} b^{T}(e+c)+H^{4} b^{T} A(e+c)-\cdots+(-1)^{s-1} H^{2 s-2} b^{T} A^{s-2}(e+c), \\
P\left(H^{2}\right)=1-H^{2} b^{T} c+H^{4} b^{T} A c-\cdots+(-1)^{s-1} H^{2 s-2} b^{T} A^{s-2} c
\end{gathered}
$$

where $e$ is the $s$-dimensional vector of units, and the vectors $c, b$, and matrix $A$ are defined by the Butcher tableau of the scheme (2.1). The polynomial

$$
\zeta^{2}-S\left(H^{2}\right) \zeta+P\left(H^{2}\right)
$$

is called the stability polynomial of the difference equation (2.4) associated with the method (2.1).

Definition 2.1. The method (2.1) is said to have a periodicity interval $I_{p}=\left(0, H_{0}^{2}\right)$ if the roots of its stability polynomial, $\xi_{1,2}(H)$ are conjugate complex and satisy $\left|\xi_{1,2}(H)\right|=1$, for all $H \in$ $\left(0, H_{0}^{2}\right)$. The method is called $P$-stable if the periodicity interval $I_{p}=(0, \infty)$.

The existence of a nonempty periodicity interval $I_{p}=\left(0, H_{0}^{2}\right)$ is equivalent to the fact that there exists a nonzero real number $H_{0}$ such that the coefficients of the stability polynomial satisfy the conditions

$$
P\left(H^{2}\right) \equiv 1, \quad\left|S\left(H^{2}\right)\right|<2, \quad \forall H \in\left(0, H_{0}^{2}\right)
$$

Therefore, the $P$-stable conditions are given by

$$
P\left(H^{2}\right) \equiv 1, \quad\left|S\left(H^{2}\right)\right|<2, \quad \forall H>0 .
$$

An interval $I_{S}=\left(0, H_{1}^{2}\right)$ is called the interval of absolute stability of the method (2.1) if (see [4])

$$
P\left(H^{2}\right)<1,\left|S\left(H^{2}\right)\right|<P\left(H^{2}\right)+1, \quad H \in\left(0, H_{1}^{2}\right) .
$$


Definition 2.2. The quantities (see [25])

$$
\phi(H)=H-\arccos \left(\frac{S\left(H^{2}\right)}{2 \sqrt{P\left(H^{2}\right)}}\right), \quad d(H)=1-\sqrt{P\left(H^{2}\right)},
$$

are called the phase-lag and the dissipation, respectively. Accordingly, the method is said to be dispersive of order $q$ and dissipative of order $p$ if $\phi(H)=\mathcal{O}\left(H^{q+1}\right)$, and $d(H)=\mathcal{O}\left(H^{p+1}\right)$, respectively. If $\phi(H)=0$, the method is said to be phase fitted or zero-dispersive, and if $d(H)=0$, the method is said to be amplification fitted or zero-dissipative.

\section{Construction of the New Methods}

In this section, we construct explicit Numerov-type methods which are both phase fitted and amplification fitted. The construction is based on the following Butcher tableau of coefficients

$$
\begin{array}{c|cccc}
-1 & 0 & 0 & 0 & 0 \\
0 & 0 & 0 & 0 & 0 \\
\frac{25}{28} & \frac{1325}{43904} & \frac{35775}{43904} & 0 & 0 \\
-\frac{23}{5} & \frac{16744}{33125} & \frac{383111}{15625} & -\frac{13866608}{828125} & 0 \\
\hline & b_{1} & b_{2} & b_{3} & b_{4}
\end{array} .
$$

If we choose $\left\{b_{1}, b_{2}, b_{3}, b_{4}\right\}=\{173 / 1908,2791 / 3450,307328 / 3056775,125 / 636732\}$, the classical Numerov-type method is recovered in [26]. In the following, we derive the frequencydependent weights $b_{i}, i=1, \ldots, 4$ of a one-frequency method and a two-frequency method by phase fitting and amplification fitting.

\subsection{A One-Frequency Numerov-Type Method}

Applying the method (3.1) to the test equation

$$
y^{\prime \prime}(x)=-\omega_{1}^{2} y(x)
$$

we obtain the following expressions for $S$ and $P$

$$
\begin{gathered}
S(u)=\frac{2-b_{2} u^{2}+53 b_{3} u^{2}\left(-1568+675 u^{2}\right)}{4390}+\frac{3 b_{4} u^{2}\left(42000-83720 u^{2}+159183 u^{4}\right)}{3500}, \\
P(u)=\frac{1+b_{1} u^{2}-25 b_{3} u^{2}\left(1568+53 u^{2}\right)}{43904}-23 b_{4} u^{2}\left(-7000+23520 u^{2}+769 u^{4}\right), \quad u=\omega_{1} h .
\end{gathered}
$$


Nullifying the dispersion $\phi(u)$ and the dissipation $d(u)$ in Definition 2.2 for the test equation (3.2) yields

$$
S(u)=2 \cos (u), \quad P(u)=1 .
$$

Choosing $b_{1}=173 / 1908, b_{2}=2791 / 3450$, we solve the linear systems (3.4) for the $b$-values as follows

$$
\begin{gathered}
b_{3}=-196\left(5119800000-19455100000 u^{2}+6757940320 u^{4}-460960901 u^{6}\right. \\
\left.+731400\left(23520 u^{2}+769 u^{4}-7000\right) \cos (u)\right) / 3056775 M u^{2} \\
b_{4}=\frac{625\left(-64915200+30302600 u^{2}-1798287 u^{4}+41400\left(1568+53 u^{2}\right) \cos (u)\right)}{636732 M u^{2}}
\end{gathered}
$$

where $M=\left(-19000+952935 u^{2}+11707 u^{4}\right)$.

It is easy to verify the above coefficients satisfy the algebraic fifth-order conditions for the Numerov-type methods with one frequency (see [23]):

$$
\begin{gathered}
b^{T} e=1-\frac{173}{940800} u^{6}+\mathcal{O}\left(u^{8}\right), \quad b^{T} c=0+\frac{1}{10080} u^{4}+\mathcal{O}\left(u^{6}\right), \\
b^{T} c^{2}=\frac{1}{6}-\frac{173}{470400} u^{2}+\mathcal{O}\left(u^{4}\right), \quad b^{T}(A e)=\frac{1}{12}-\frac{379}{2822400} u^{2}+\mathcal{O}\left(u^{4}\right), \\
b^{T} c^{3}=0+\frac{349861}{197568000} u^{2}+\mathcal{O}\left(u^{4}\right), \quad b^{T}(c \cdot(A e))=\frac{1}{12}-\frac{277201}{395136000} u^{2}+\mathcal{O}\left(u^{4}\right), \\
b^{T}(A c)=0+\frac{110087}{395136000} u^{2}+\mathcal{O}\left(u^{4}\right), \quad b^{T} c^{4}=\frac{1}{15}-\frac{74452453}{9219840000} u^{2}+\mathcal{O}\left(u^{4}\right), \\
b^{T}\left(c^{2} \cdot A e\right)=\frac{1}{30}-\frac{174376819}{55319040000} u^{2}+\mathcal{O}\left(u^{4}\right), \quad b^{T}(c \cdot(A c))=-\frac{1}{60}-\frac{71061653}{55319040000} u^{2}+\mathcal{O}\left(u^{4}\right), \\
b^{T}(A e \cdot A e)=\frac{7}{120}-\frac{135568679}{110638080000} u^{2}+\mathcal{O}\left(u^{4}\right), \quad b^{T}\left(A c^{2}\right)=\frac{1}{180}+\frac{153119}{658560000} u^{2}+\mathcal{O}\left(u^{4}\right), \\
b^{T}\left(A^{2} e\right)=\frac{1}{360}+\frac{23}{90000} u^{2}+\mathcal{O}\left(u^{4}\right) .
\end{gathered}
$$

This one-frequency phase fitted and amplification fitted fifth-order method is denoted as PFAFI.

\subsection{A Two-Frequency Numerov-Type Method}

For the method (3.1), we only need the weights $b_{i}(i=1, \ldots, 4)$. In order to solve the oscillatory problems with two frequencies, we consider another test equation

$$
y^{\prime \prime}(x)=-\omega_{2}^{2} y(x), \quad \omega_{2} \neq \omega_{1}
$$


With the same idea stated above, we find the following relation

$$
S(v)=2 \cos (v), \quad P(v)=1, \quad v=\omega_{2} h
$$

Solving (3.4) and (3.8) gives

$$
\begin{aligned}
b_{1}= & \frac{\left(56\left(865+28 v^{2}\right)+u^{2}\left(1568+53 v^{2}\right)\right)\left(u^{2}-v^{2}+v^{2} \cos (u)-u^{2} \cos (v)\right)}{M}, \\
b_{2}=( & -1107120\left(u^{2}-v^{2}\right)+446936\left(u^{4}-v^{4}\right)+15525 u^{2} v^{2}\left(u^{2}-v^{2}\right) \\
& +v^{2}\left(23 u^{2}\left(-1568+675 v^{2}\right)+56\left(-19770+7981 v^{2}\right)\right) \cos (u) \\
& \left.-u^{2}\left(23 u^{2}\left(19432+675 v^{2}\right)-112\left(9885+322 v^{2}\right)\right) \cos (v)\right) / N, \\
b_{3}= & \frac{-1568\left(23520+769\left(u^{2}+v^{2}\right)\right)\left(v^{2}-u^{2}-v^{2} \cos (u)+u^{2} \cos (v)\right)}{T}, \\
b_{4}= & \frac{250\left(v^{2}-u^{2}-v^{2} \cos (u)+u^{2} \cos (v)\right)}{53061 u^{2} v^{2}\left(u^{2}-v^{2}\right)} .
\end{aligned}
$$

In which $M=22260 u^{2} v^{2}\left(u^{2}-v^{2}\right), N=241500 u^{2} v^{2}\left(u^{2}-v^{2}\right), T=15283875 u^{2} v^{2}\left(u^{2}-v^{2}\right)$.

It is also easy to verify the algebraic fifth order conditions for the Numerov-type methods with two frequencies (see [23] for reference)

$$
\begin{gathered}
b^{T} e=1-\frac{13\left(\omega_{1}^{2} \omega_{2}^{2}\left(\omega_{1}^{2}+\omega_{2}^{2}\right)\right) h^{6}}{302400}+\mathcal{O}\left(h^{8}\right), \quad b^{T} c=0-\frac{\left(\omega_{1}^{2} \omega_{2}^{2}\right) h^{4}}{10080}+\mathcal{O}\left(h^{6}\right), \\
b^{T} c^{2}=\frac{1}{6}-\frac{13\left(\omega_{1}^{2}+\omega_{2}^{2}\right)^{2} h^{4}}{151200}+\mathcal{O}\left(h^{6}\right), \quad b^{T}(A e)=\frac{1}{12}-\frac{\left(13 \omega_{1}^{4}+41 \omega_{1}^{2} \omega_{2}^{2}+13 \omega_{2}^{4}\right) h^{4}}{302400}+\mathcal{O}\left(h^{6}\right), \\
b^{T} c^{3}=0-\frac{\left(\omega_{1}^{2}+\omega_{2}^{2}\right) h^{2}}{1680}+\mathcal{O}\left(h^{4}\right), \quad b^{T}(c \cdot(A e))=\frac{1}{12}-\frac{\left(\omega_{1}^{2}+\omega_{2}^{2}\right) h^{2}}{3360}+\mathcal{O}\left(h^{4}\right), \\
b^{T}(A c)=0-\frac{\left(\omega_{1}^{2}+\omega_{2}^{2}\right) h^{2}}{10080}+\mathcal{O}\left(h^{4}\right), \quad b^{T} c^{4}=\frac{1}{15}+\frac{659\left(\omega_{1}^{2}+\omega_{2}^{2}\right) h^{2}}{235200}+\mathcal{O}\left(h^{4}\right), \\
b^{T}\left(c^{2} \cdot A e\right)=\frac{1}{30}+\frac{173\left(\omega_{1}^{2}+\omega_{2}^{2}\right) h^{2}}{156800}+\mathcal{O}\left(h^{4}\right), \quad b^{T}(c \cdot(A c))=-\frac{1}{60}+\frac{659\left(\omega_{1}^{2}+\omega_{2}^{2}\right) h^{2}}{1411200}+\mathcal{O}\left(h^{4}\right), \\
b^{T}(A e \cdot A e)=\frac{7}{120}+\frac{379\left(\omega_{1}^{2}+\omega_{2}^{2}\right) h^{2}}{940800}+\mathcal{O}\left(h^{4}\right), \quad b^{T}\left(A c^{2}\right)=\frac{1}{180}-\frac{13\left(\omega_{1}^{2}+\omega_{2}^{2}\right) h^{2}}{151200}+\mathcal{O}\left(h^{4}\right), \\
b^{T}\left(A^{2} e\right)=\frac{1}{360}-\frac{\left(\omega_{1}^{2}+\omega_{2}^{2}\right) h^{2}}{10800}+\mathcal{O}\left(h^{4}\right) .
\end{gathered}
$$

The two-frequency fifth-order Numerov-type method with the weights in (3.9) is denoted as PFAFII. 


\section{Stability and Phase Analysis}

In this section, we analyze the stability and phase properties of the two new methods derived in Section 3. The theory of Lambert and Watson was reconsidered by Coleman and Ixaru [27].

\subsection{Stability and Phase Analysis of PFAFI}

We apply a four-stage one-frequency Numerov-type method to the test equation (2.3) and obtain the functions $P$ and $S$ in (2.4) as follows

$$
\begin{gathered}
P\left(H^{2}, u\right)=1-H^{2} b(u)^{T} c+H^{4} b(u)^{T} A c-H^{6} b(u)^{T} A^{2} c \\
S\left(H^{2}, u\right)=2-H^{2} b(u)^{T}(e+c)+H^{4} b(u)^{T} A(e+c)-H^{6} b(u)^{T} A^{2}(e+c) .
\end{gathered}
$$

The stability and the phase properties depend on the bivariate functions $S\left(H^{2}, u\right)$ and $P\left(H^{2}, u\right)$. In this situation, the interval of absolute stability is replaced by a two-dimensional region.

Definition 4.1. For the Numerov-type method with $S\left(H^{2}, u\right)$ and $P\left(H^{2}, u\right)$ given by $(4.1)$, the quantities

$$
\phi(H, u)=H-\arccos \left(\frac{S\left(H^{2}, u\right)}{2 \sqrt{P\left(H^{2}, u\right)}}\right), \quad d(H, u)=1-\sqrt{P\left(H^{2}, u\right)}
$$

are called the phase-lag and the dissipation, respectively. If

$$
\phi(H, u)=\mathcal{O}\left(H^{q+1}\right), \quad d(H, u)=\mathcal{O}\left(H^{p+1}\right)
$$

then the method is said to be dissipative of order $q$ and dissipative of order $p$, respectively.

For convenience of analyzing the phase-lag and the dissipation, we denote the ratio $r=u / H$. Then the phase-lag and the dissipation of the new method PFAFI are given by

$$
\begin{gathered}
\phi=-\frac{13\left(r^{2}\left(r^{2}-1\right)\right)}{604800} H^{7}+\mathcal{O}\left(H^{9}\right), \\
d=\frac{r^{2}-1}{20160} H^{6}+\mathcal{O}\left(H^{8}\right) .
\end{gathered}
$$

Thus the method PFAFI is dispersive of order six and dissipative of order five.

Definition 4.2. In the $H-u$ plane, the region

$$
\Omega=\left\{(H, u)\left|H>0, u>0, P\left(H^{2}, u\right)<1,\right| S\left(H^{2}, u\right) \mid<P\left(H^{2}, u\right)+1\right\}
$$




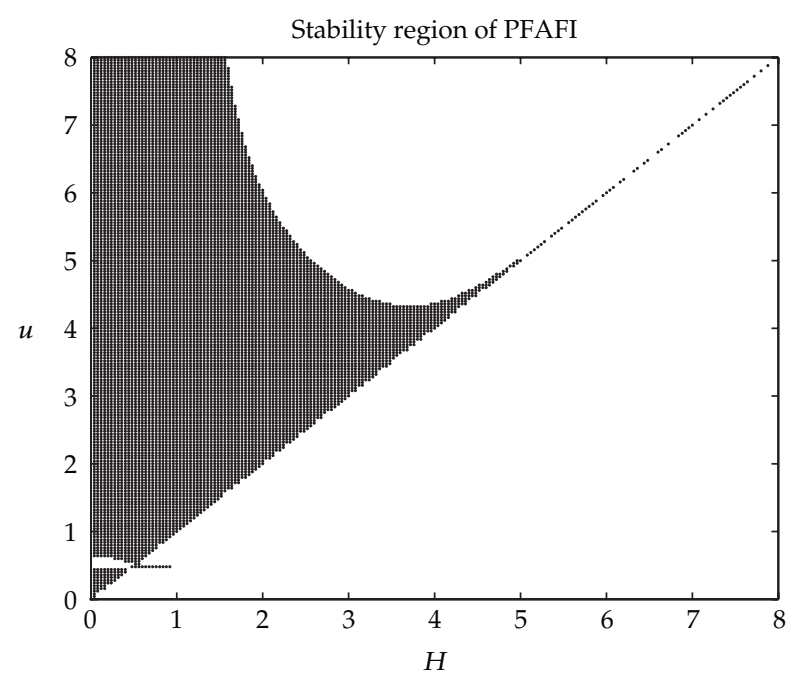

Figure 1: Stability region for the method PFAFI.

is said to be the region of absolute stability of the Numerov-type method PFAFI. Any closed curve defined by

$$
P\left(H^{2}, u\right)=1 \text { or }\left|S\left(H^{2}, u\right)\right|=P\left(H^{2}, u\right)+1
$$

is a stability boundary of the method.

In Figure 1, we plot the region of the absolute stability of the new method PFAFI derived in Section 3.1 (a similar discussion can be found in [27, 28]).

It should be noted that when the exact value of the frequency of the problem is known (i.e., the fitted frequency $\omega$ equals the test frequency $\lambda$ ), then $u=H$ and we have that $S\left(H^{2}, H\right)=2 \cos (H)$ and $P\left(H^{2}, H\right)=1$. Consequently, when the main frequency is known the interval of periodicity is $(0, \infty)$ except for $H=2 \pi, 4 \pi, \ldots$. So the new method PFAFI is almost $P$-stable.

\subsection{Stability and Phase Analysis of Phase Fitted Numerov-Type Method with Two Frequencies}

We apply a four-stage two-frequency Numerov-type method to the test equation (2.3) and obtain the functions $P$ and $S$ in (2.4) as follows

$$
\begin{gathered}
P\left(H^{2}, u, v\right)=1-H^{2} b(u, v)^{T} c+H^{4} b(u, v)^{T} A c-H^{6} b(u, v)^{T} A^{2} c \\
S\left(H^{2}, u, v\right)=2-H^{2} b(u, v)^{T}(e+c)+H^{4} b(u, v)^{T} A(e+c)-H^{6} b(u, v)^{T} A^{2}(e+c) .
\end{gathered}
$$


Definition 4.3. For the Numerov-type method with $S\left(H^{2}, u, v\right)$ and $P\left(H^{2}, u, v\right)$ given by (4.7), the quantities

$$
\phi(H, u, v)=H-\arccos \left(\frac{S\left(H^{2}, u, v\right)}{2 \sqrt{P\left(H^{2}, u, v\right)}}\right), \quad d(H, u, v)=1-\sqrt{P\left(H^{2}, u, v\right)}
$$

are called the phase-lag and the dissipation, respectively. If

$$
\phi(H, u, v)=\mathcal{O}\left(H^{q+1}\right), \quad d(H, u, v)=\mathcal{O}\left(H^{p+1}\right),
$$

then this method is said to be dispersive of order $q$ and dissipative of order $p$, respectively.

Denote $r=u / H$ and $k=v / u$. Then the phase-lag and the dissipation of the new method PFAFII are given by

$$
\begin{gathered}
\phi=\frac{13 r^{2}\left(r^{2}-1\right)\left(1+k^{2}\right)\left(k^{2} r^{2}-1\right)}{604800} H^{7}+\mathcal{O}\left(H^{9}\right), \\
d=-\frac{\left(r^{2}-1\right)\left(r^{2} k^{2}-1\right)}{20160} H^{6}+\mathcal{O}\left(H^{8}\right) .
\end{gathered}
$$

Thus the new method PFAFII is dispersive of order six and dissipative of order five.

Following the main idea of Coleman and Ixaru in [27], the absolute stability of the two-frequency method PFAFII can be described by a three-dimensional region.

Definition 4.4. A three-dimensional region $\Omega$ in the $H-u-v$ space

$$
V=\left\{(H, u, v)\left|P\left(H^{2}, u, v\right)<1,\right| S\left(H^{2}, u, v\right) \mid<P\left(H^{2}, u, v\right)+1\right\}
$$

is called the region of absolute stability of a Numerov-type method with the $S\left(H^{2}, u, v\right)$ and $P\left(H^{2}, u, v\right)$ given by (4.7), and any closed curve defined by

$$
P\left(H^{2}, u, v\right)=1 \text { or }\left|S\left(H^{2}, u, v\right)\right|=P\left(H^{2}, u, v\right)+1
$$

is a stability boundary of the method.

In Figure 2, we plot the region of absolute stability for the method PFAFII, and Figure 3 shows the projection of the three-dimensional region onto the $u$ - $H$ plane. (A similar discussion can be found in $[22,29]$.)

It should be noted that, for the new method PFAFII and for a problem with one frequency, when the exact value of the frequency of the problem is known, that is, $\omega_{1}=$ $\omega_{2}=\lambda$, then $u=v=H$ and we have that $S\left(H^{2}, H, H\right)=2 \cos (H)$ and $P\left(H^{2}, H, H\right)=1$. Consequently, the interval of periodicity is $(0, \infty)$ with the points $H=2 \pi, 4 \pi, \ldots$ deleted. That is, the new method PFAFII is also almost $P$-stable. 


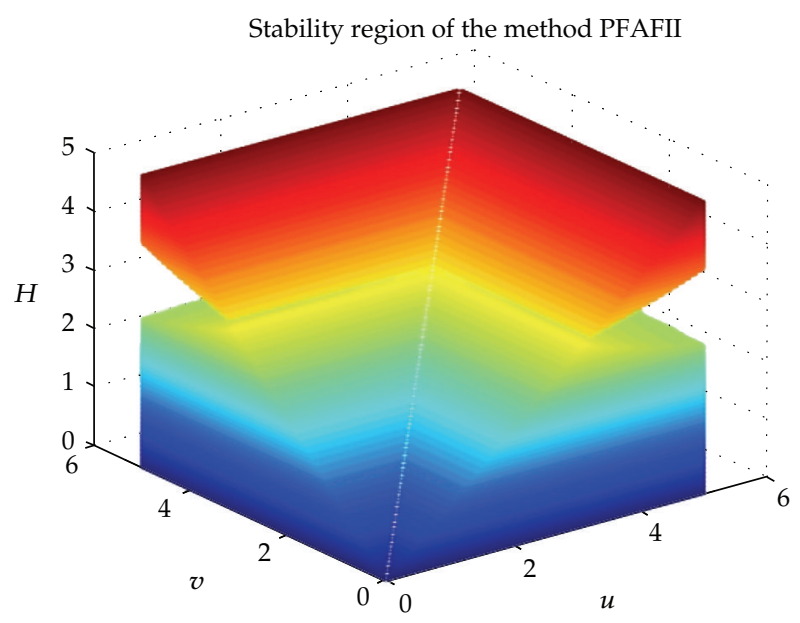

Figure 2: Stability region for the method PFAFII.

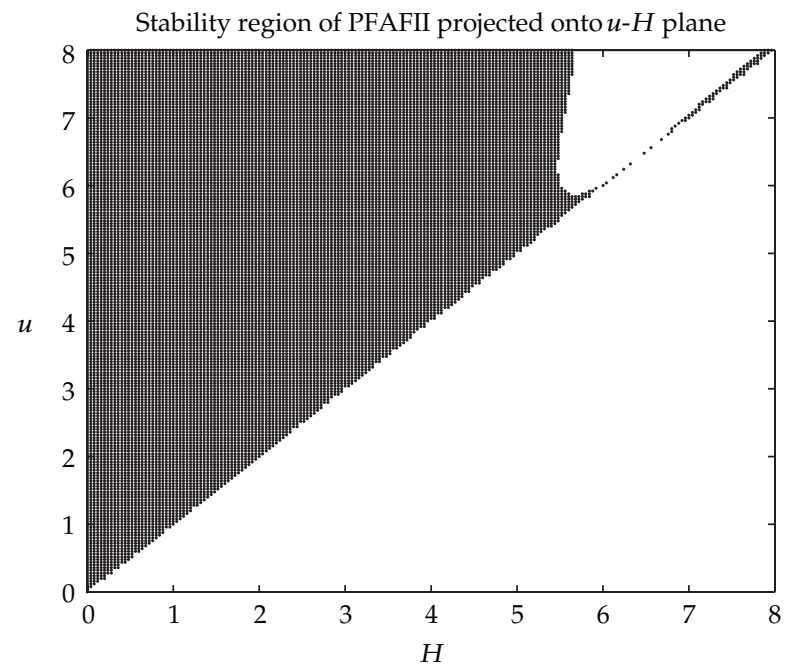

Figure 3: The projection of the three-dimensional region of PFAFII on the $u$ - $H$ plane.

\section{Numerical Experiments}

In this section, we compare the numerical performance of the new methods with some wellknown methods from the literature. We select three periodic IVPs with two frequencies. The methods we choose for comparison are listed as follows.

(i) EFRKN4F: the trigonometrically fitted RKN method of order 4 with the FSAL property given in [2].

(ii) VPHA: the phase fitted and amplification fitted Numerov-type method with one frequency given in [4].

(iii) TFNTONE: the trigonometrically fitted Numerov-type method obtained by Fang and $\mathrm{Wu}$ in [3]. 
Table 1: Comparison of the end-point global errors and the consumed CPU time in seconds (in the brackets) produced by the five methods for Problem 1.

\begin{tabular}{lccccc}
\hline$h$ & EFRKN4F & VPHA & TFNTONE & PFAFI & PFAFII \\
\hline 1 & NaN $(0.09)$ & $674.5(0.08)$ & $63.2(0.06)$ & $0.27(0.06)$ & $7.9 e-3(0.06)$ \\
$1 / 2$ & $0.17(1.19)$ & $12.7(0.13)$ & $1.99(0.11)$ & $0.04(0.13)$ & $1.3 e-3(0.11)$ \\
$1 / 4$ & $4.6 e-3(0.34)$ & $2.3 e-2(0.28)$ & $2.7 e-3(0.23)$ & $1.5 e-4(0.23)$ & $1.1 e-5(0.23)$ \\
$1 / 8$ & $1.5 e-4(0.71)$ & $2.4 e-4(0.53)$ & $2.8 e-5(0.47)$ & $2.3 e-6(0.48)$ & $1.9 e-7(0.48)$ \\
$1 / 16$ & $0.7 e-6(1.39)$ & $3.4 e-6(1.13)$ & $1.43 e-7(0.95)$ & $1.3 \times 10^{-7}(0.95)$ & $4.9 e-9(0.95)$ \\
\hline
\end{tabular}

(iv) PFAFI: the phase fitted and amplification fitted Numerov-type method with one frequency obtained in this paper.

(v) PFAFII: the phase fitted and amplification fitted Numerov-type method with two frequencies obtained in this paper.

Problem 1. We consider the inhomogeneous equation

$$
y^{\prime \prime}(x)+100 y(x)=99 \sin (x), \quad y(0)=1, \quad y^{\prime}(0)=11,
$$

whose exact solution is given by

$$
y(x)=\cos (10 x)+\sin (10 x)+\sin (x) .
$$

In our test we choose $\omega_{1}=10$ for VPHA, TFNTONE, EFRKN4F and PFAFI, and $\omega_{1}=10, \omega_{2}=$ 1 for PFAFII. The problem is integrated on the interval $[0,1000]$ and the numerical results are presented in Table 1.

Problem 2. Consider the famous Kramarz system (see [30])

$$
\begin{array}{ccc}
y^{\prime \prime}=2498 y+4998 z, & y(0)=2, & y^{\prime}(0)=0, \\
z^{\prime \prime}=-2499 y-4999 z, & z(0)=-1, & z^{\prime}(0)=0,
\end{array}
$$

whose exact solution is

$$
y(x)=2 \cos (x), \quad z(x)=-\cos (x)
$$

The eigenvalues of the matrix of coefficients of the equations for $y^{\prime \prime}$ and $z^{\prime \prime}$ are -1 and -2500 . For this stiff equation, we choose $\omega_{1}=1$ for VPHA, TFNTONE, EFRKN4F and PFAFI, and $\omega_{1}=50, \omega_{2}=1$ for PFAFII. The problem is integrated on the interval $[0,1000]$ and the numerical results are presented in Table 2. 
Table 2: Comparison of the end-point global errors and the consumed CPU time in seconds (in the brackets) produced by the five methods for Problem 2 .

\begin{tabular}{lccccc}
\hline$h$ & EFRKN4F & VPHA & TFNTONE & PFAFI & PFAFII \\
\hline 8 & $\mathrm{NaN}(0.02)$ & $\mathrm{NaN}(0.03)$ & $\mathrm{NaN}(0.02)$ & $\mathrm{NaN}(0.02)$ & $6.7 e-12(0.02)$ \\
4 & $\mathrm{NaN}(0.05)$ & $\mathrm{NaN}(0.05)$ & $\mathrm{NaN}(0.03)$ & $\mathrm{NaN}(0.03)$ & $2.5 e-12(0.03)$ \\
2 & $\mathrm{NaN}(0.09)$ & $\mathrm{NaN}(0.09)$ & $\mathrm{NaN}(0.06)$ & $\mathrm{NaN}(0.05)$ & $2.0 e-11(0.06)$ \\
1 & $\mathrm{NaN}(0.17)$ & $\mathrm{NaN}(0.19)$ & $\mathrm{NaN}(0.13)$ & $\mathrm{NaN}(0.11)$ & $1.8 e-11(0.09)$ \\
$1 / 2$ & $\mathrm{NaN}(0.34)$ & $\mathrm{NaN}(0.38)$ & $\mathrm{NaN}(0.25)$ & $\mathrm{NaN}(0.23)$ & $1.0 e-11(0.20)$ \\
$1 / 4$ & $\mathrm{NaN}(0.70)$ & $\mathrm{NaN}(0.77)$ & $\mathrm{NaN}(0.5)$ & $\mathrm{NaN}(0.47)$ & $8.0 e-12(0.40)$ \\
$1 / 8$ & $\mathrm{NaN}(1.42)$ & $\mathrm{NaN}(1.53)$ & $\mathrm{NaN}(1.0)$ & $\mathrm{NaN}(0.92)$ & $1.7 e-11(0.82)$ \\
$1 / 16$ & $1.2 e-12(2.56)$ & $1.6 e-11(2.70)$ & $2.3 e-12(1.75)$ & $\mathrm{NaN}(1.86)$ & $1.8 e-11(1.65)$ \\
\hline
\end{tabular}

Table 3: Comparison of the end-point global errors and the consumed CPU time in seconds (in the brackets) produced by the five methods for Problem 3 with $\epsilon=10^{-7}, \omega=10^{3}$.

\begin{tabular}{lccccc}
\hline$h$ & EFRKN4F & VPHA & TFNTONE & PFAFI & PFAFII \\
\hline 2 & $\mathrm{NaN}(0)$ & $\mathrm{NaN}(0)$ & $\mathrm{NaN}(0)$ & $\mathrm{NaN}(0)$ & $2.6 e-11(0)$ \\
1 & $\mathrm{NaN}(0)$ & $\mathrm{NaN}(0)$ & $0.01(0)$ & $\mathrm{NaN}(0)$ & $3.0 e-11(0)$ \\
$1 / 2$ & $\mathrm{NaN}(0.015)$ & $\mathrm{NaN}(0.015)$ & $0.35(0.015)$ & $1.38(0.015)$ & $2.9 e-12(0.015)$ \\
$1 / 4$ & $\mathrm{NaN}(0.016)$ & $\mathrm{NaN}(0.016)$ & $0.025(0.016)$ & $1.43(0.016)$ & $4.7 e-11(0.016)$ \\
$1 / 8$ & $\mathrm{NaN}(0.016)$ & $\mathrm{NaN}(0.016)$ & $3.3 e-3(0.016)$ & $1.56(0.016)$ & $1.4 e-11(0.015)$ \\
$1 / 16$ & $\mathrm{NaN}(0.031)$ & $\mathrm{NaN}(0.016)$ & $2.6 e-3(0.016)$ & $1.68(0.016)$ & $1.5 e-11(0.031)$ \\
$1 / 32$ & $\mathrm{NaN}(0.047)$ & $\mathrm{NaN}(0.031)$ & $8.6 e-4(0.031)$ & $1.42(0.031)$ & $1.3 e-12(0.031)$ \\
\hline
\end{tabular}

Problem 3. Consider the linear stiff system in [31]

$$
\begin{gathered}
y^{\prime \prime}(t)=-\frac{1}{2}\left(\begin{array}{cc}
\omega^{2}+1 & \omega^{2}-1 \\
\omega^{2}-1 & \omega^{2}+1
\end{array}\right) y(t), \\
y(0)=(1+\epsilon, \epsilon-1)^{T}, \quad y^{\prime}(0)=(\epsilon \omega+1, \epsilon \omega-1)^{T},
\end{gathered}
$$

whose analytic solution is given by

$$
y(t)=\left(\begin{array}{c}
\cos (t)+\sin (t) \\
-\cos (t)-\sin (t)
\end{array}\right)+\epsilon\left(\begin{array}{c}
\cos (\omega t)+\sin (\omega t) \\
\cos (\omega t)+\sin (\omega t)
\end{array}\right)
$$

In our test we choose $\omega_{1}=\omega$ for VPHA, TFNTONE, EFRKN4F and PFAFI, and $\omega_{1}=\omega, \omega_{2}=1$ for PFAFII. The problem is integrated on the interval $[0,10]$ and the numerical results are presented in Table 3 with $\epsilon=10^{-7}, \omega=10^{3}$, and in Table 4 with $\epsilon=10^{-7}, \omega=10^{5}$.

Problem 4. Consider the nonlinear system in [32]

$$
\begin{gathered}
y^{\prime \prime}(t)=\left(\begin{array}{cc}
-1 & 0 \\
0 & -\lambda^{2}
\end{array}\right) y(t)+k\left(\begin{array}{l}
1 \\
1
\end{array}\right)\left(\|y(t)\|_{2}^{2}+\cos ^{2}(t)-1\right), \\
y(0)=(0,0)^{T}, \quad y^{\prime}(0)=(1,0)^{T},
\end{gathered}
$$


Table 4: Comparison of the end-point global errors and the consumed CPU time in seconds (in the brackets) produced by the five methods for Problem 3 with $\epsilon=10^{-7}, \omega=10^{5}$.

\begin{tabular}{|c|c|c|c|c|c|}
\hline$h$ & EFRKN4F & VPHA & TFNTONE & PFAFI & PFAFII \\
\hline $1 / 8$ & $\mathrm{NaN}(0.02)$ & $\mathrm{NaN}(0.02)$ & $0.29(0.01)$ & $\mathrm{NaN}(0.01)$ & $5.7 e-7(0.02)$ \\
\hline $1 / 16$ & $\mathrm{NaN}(0.03)$ & $\mathrm{NaN}(0.03)$ & $0.05(0.02)$ & $1.76(0.02)$ & $8.3 e-8(0.03)$ \\
\hline $1 / 32$ & $\mathrm{NaN}(0.06)$ & $\mathrm{NaN}(0.06)$ & $8.8 e-3(0.03)$ & $1.91(0.03)$ & $7.5 e-8(0.05)$ \\
\hline $1 / 64$ & $\mathrm{NaN}(0.11)$ & $\mathrm{NaN}(0.11)$ & $8.6 e-3(0.06)$ & $2.01(0.05)$ & $4.1 e-8(0.08)$ \\
\hline $1 / 128$ & $\mathrm{NaN}(0.20)$ & $\mathrm{NaN}(0.23)$ & $1.8 e-3(0.14)$ & $2.07(0.08)$ & $3.3 e-8(0.14)$ \\
\hline $1 / 256$ & $\mathrm{NaN}(0.43)$ & $\mathrm{NaN}(0.45)$ & $9.0 e-5(0.27)$ & $2.10(0.17)$ & $1.1 e-8(0.27)$ \\
\hline $1 / 512$ & $\mathrm{NaN}(0.89)$ & $\mathrm{NaN}(0.93)$ & $1.8 e-6(0.53)$ & $2.11(0.36)$ & $4.6 e-9(0.53)$ \\
\hline
\end{tabular}

Table 5: Comparison of the end-point global errors and the consumed CPU time in seconds (in the brackets) produced by the five methods for Problem 4 .

\begin{tabular}{|c|c|c|c|c|c|}
\hline$h$ & EFRKN4F & VPHA & TFNTONE & PFAFI & PFAFII \\
\hline $1 / 4$ & $\mathrm{NaN}(0.76)$ & $\mathrm{NaN}(0.61)$ & $\mathrm{NaN}(0.53)$ & $\mathrm{NaN}(0.42)$ & $3.45 e-7(0.43)$ \\
\hline $1 / 8$ & $\mathrm{NaN}(1.52)$ & $\mathrm{NaN}(1.24)$ & $\mathrm{NaN}(0.89)$ & $\mathrm{NaN}(0.81)$ & $2.14 e-8(0.89)$ \\
\hline $1 / 16$ & $\mathrm{NaN}(3.06)$ & $\mathrm{NaN}(2.45)$ & $\mathrm{NaN}(1.79)$ & $\mathrm{NaN}(1.60)$ & $1.33 e-9(1.78)$ \\
\hline $1 / 32$ & $\mathrm{NaN}(6.04)$ & $\mathrm{NaN}(4.89)$ & $0.008(3.56)$ & $0.82(2.5)$ & $3.98 e-11(3.59)$ \\
\hline $1 / 64$ & $\mathrm{NaN}(12.07)$ & $\mathrm{NaN}(9.73)$ & $0.08(7.1)$ & $0.82(5)$ & $4.39 e-12(7.1)$ \\
\hline
\end{tabular}

where $\lambda=10^{3}, k=0.01$. The analytic solution of the problem is

$$
y(t)=(\sin (t), 0)^{T} .
$$

In our test we choose $\omega=\lambda$ for VPHA, TFNTONE, EFRKN4F and PFAFI, and $\omega_{1}=$ $\lambda, \omega_{2}=1$ for PFAFII. The problem is integrated on the integral interval $[0,1000]$ and the numerical results are stated in Table 5.

\section{Conclusions}

In this paper, we investigate Numerov-type methods adapted to oscillatory problems with two frequencies obtained by phase fitting and amplification fitting. The numerical stability and the phase properties of the new method are analyzed. Unlike the case of one-frequency methods, the absolute stability region of a two-frequency method is a region in the threedimensional space. The results of numerical experiments on four oscillatory test problems with two frequencies, including non-stiff and stiff problems, show that the new methods are superior to some well-known high quality codes proposed in the recent scientific literature.

\section{Acknowledgments}

This research was partially supported by NSFC (no. 11101357, no. 11171155), the foundation of Shandong Outstanding Young Scientists Award Project (no. BS2010SF031), the foundation of Scientific Research Project of Shandong Universities (no. J11LG69), NSF of Shandong Province, China (no. ZR2011AL006) and the Fundamental Research Fund for the Central Universities (no. Y0201100265, no. KYZ201125). 


\section{References}

[1] G. Vanden Berghe, H. De Meyer, M. Van Daele, and T. Van Hecke, "Exponentially-fitted explicit Runge-Kutta methods," Computer Physics Communications, vol. 123, no. 1-3, pp. 7-15, 1999.

[2] J. M. Franco, "Exponentially fitted explicit Runge-Kutta-Nyström methods," Journal of Computational and Applied Mathematics, vol. 167, no. 1, pp. 1-19, 2004.

[3] Y. L. Fang and X. Y. Wu, "A trigonometrically fitted explicit Numerov-type method for second-order initial value problems with oscillating solutions," Applied Numerical Mathematics, vol. 58, no. 3, pp. 341-351, 2008.

[4] H. Van de Vyver, "An explicit Numerov-type method for second-order differential equations with oscillating solutions," Computers \& Mathematics with Applications, vol. 53, no. 9, pp. 1339-1348, 2007.

[5] A. Konguetsof and T. E. Simos, "A generator of hybrid symmetric four-step methods for the numerical solution of the Schrödinger equation," Journal of Computational and Applied Mathematics, vol. 158, no. 1, pp. 93-106, 2003.

[6] Z. Kalogiratou, Th. Monovasilis, and T. E. Simos, "Symplectic integrators for the numerical solution of the Schrödinger equation," Journal of Computational and Applied Mathematics, vol. 158, no. 1, pp. 83-92, 2003.

[7] Z. Kalogiratou and T. E. Simos, "Newton-Cotes formulae for long-time integration," Journal of Computational and Applied Mathematics, vol. 158, no. 1, pp. 75-82, 2003.

[8] G. Psihoyios and T. E. Simos, "Trigonometrically fitted predictor-corrector methods for IVPs with oscillating solutions," Journal of Computational and Applied Mathematics, vol. 158, no. 1, pp. 135-144, 2003.

[9] T. E. Simos, I. T. Famelis, and C. Tsitouras, "Zero dissipative, explicit Numerov-type methods for second order IVPs with oscillating solutions," Numerical Algorithms, vol. 34, no. 1, pp. 27-40, 2003.

[10] T. E. Simos, "Dissipative trigonometrically-fitted methods for linear second-order IVPs with oscillating solution," Applied Mathematics Letters, vol. 17, no. 5, pp. 601-607, 2004.

[11] K. Tselios and T. E. Simos, "Runge-Kutta methods with minimal dispersion and dissipation for problems arising from computational acoustics," Journal of Computational and Applied Mathematics, vol. 175, no. 1, pp. 173-181, 2005.

[12] D. P. Sakas and T. E. Simos, "Multiderivative methods of eighth algebraic order with minimal phaselag for the numerical solution of the radial Schrödinger equation," Journal of Computational and Applied Mathematics, vol. 175, no. 1, pp. 161-172, 2005.

[13] G. Psihoyios and T. E. Simos, "A fourth algebraic order trigonometrically fitted predictor-corrector scheme for IVPs with oscillating solutions," Journal of Computational and Applied Mathematics, vol. 175, no. 1, pp. 137-147, 2005.

[14] Z. A. Anastassi and T. E. Simos, "An optimized Runge-Kutta method for the solution of orbital problems," Journal of Computational and Applied Mathematics, vol. 175, no. 1, pp. 1-9, 2005.

[15] T. E. Simos, "Closed Newton-Cotes trigonometrically-fitted formulae of high order for long-time integration of orbital problems," Applied Mathematics Letters, vol. 22, no. 10, pp. 1616-1621, 2009.

[16] S. Stavroyiannis and T. E. Simos, "Optimization as a function of the phase-lag order of nonlinear explicit two-step P-stable method for linear periodic IVPs," Applied Numerical Mathematics, vol. 59, no. 10, pp. $2467-2474,2009$.

[17] T. E. Simos, "Exponentially and trigonometrically fitted methods for the solution of the Schrödinger equation," Acta Applicandae Mathematicae, vol. 110, no. 3, pp. 1331-1352, 2010.

[18] T. E. Simos, "New stable closed Newton-Cotes trigonometrically fitted formulae for long-time integration," Abstract and Applied Analysis, vol. 2012, Article ID 182536, 15 pages, 2012.

[19] T. E. Simos, "Optimizing a hybrid two-step method for the numerical solution of the Schrödinger equation and related problems with respect to phase-lag," Journal of Applied Mathematics, vol. 2012, Article ID 420387, 17 pages, 2012.

[20] Z.A. Anastassi and T.E. Simos, "A parametric symmetric linear four-step method for the efficient integration of the Schrödinger equation and related oscillatory problems," Journal of Computational and Applied Mathematics, vol. 236, no. 16, pp. 3880-3889, 2012.

[21] Z. C. Wang, "Trigonometrically-fitted method for a periodic initial value problem with two frequencies," Computer Physics Communications, vol. 175, pp. 241-249, 2006.

[22] Y. L. Fang, Y. Z. Song, and X. Y. Wu, "Trigonometrically fitted explicit Numerov-type method for periodic IVPs with two frequencies," Computer Physics Communications, vol. 179, no. 11, pp. 801-811, 2008. 
[23] J. P. Coleman, "Order conditions for a class of two-step methods for $y^{\prime \prime}=f(x, y)$," IMA Journal of Numerical Analysis, vol. 23, no. 2, pp. 197-220, 2003.

[24] J. D. Lambert and I. A. Watson, "Symmetric multistep methods for periodic initial value problems," Journal of the Institute of Mathematics and its Applications, vol. 18, no. 2, pp. 189-202, 1976.

[25] P. J. van der Houwen and B. P. Sommeijer, "Explicit Runge-Kutta (-Nyström) methods with reduced phase errors for computing oscillating solutions," SIAM Journal on Numerical Analysis, vol. 24, no. 3, pp. 595-617, 1987.

[26] J. M. Franco, "A class of explicit two-step hybrid methods for second-order IVPs," Journal of Computational and Applied Mathematics, vol. 187, no. 1, pp. 41-57, 2006.

[27] J. P. Coleman and L. Gr. Ixaru, "P-stability and exponential-fitting methods for $y^{\prime \prime}=f(x, y)$," IMA Journal of Numerical Analysis, vol. 16, no. 2, pp. 179-199, 1996.

[28] H. Van de Vyver, "Stability and phase-lag analysis of explicit Runge-Kutta methods with variable coefficients for oscillatory problems," Computer Physics Communications, vol. 173, no. 3, pp. 115-130, 2005.

[29] R. D'Ambrosio, M. Ferro, and B. Paternoster, "Trigonometrically fitted two-step hybrid methods for special second order ordinary differential equations," Mathematics and Computers in Simulation, vol. 81, no. 5, pp. 1068-1084, 2011.

[30] L. Kramarz, "Stability of collocation methods for the numerical solution of $y^{\prime \prime}=f(x, y)$," BIT; Nordisk Tidskrift for Informationsbehandling (BIT), vol. 20, no. 2, pp. 215-222, 1980.

[31] J. M. Franco and I. Gómez, "Accuracy and linear stability of RKN methods for solving second-order stiff problems," Applied Numerical Mathematics, vol. 59, no. 5, pp. 959-975, 2009.

[32] J. M. Franco, I. Gómez, and L. Rández, "Four-stage symplectic and P-stable SDIRKN methods with dispersion of high order," Numerical Algorithms, vol. 26, no. 4, pp. 347-363, 2001. 


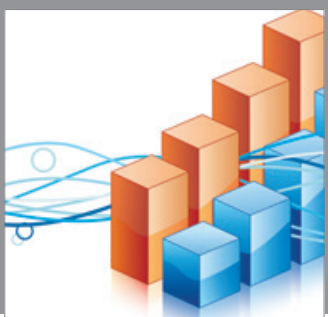

Advances in

Operations Research

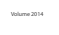

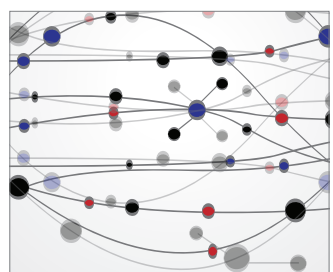

\section{The Scientific} World Journal
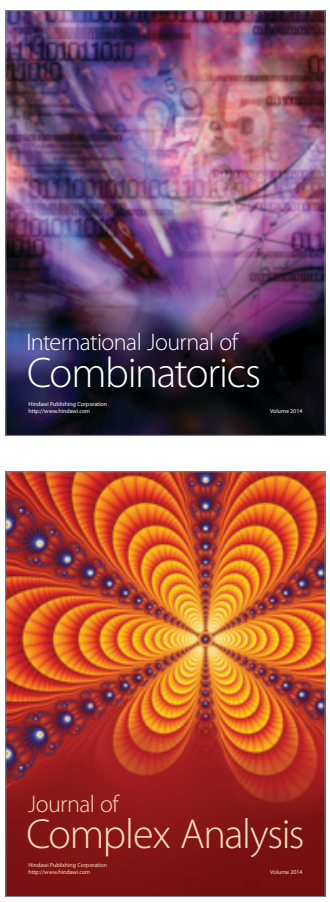

International Journal of

Mathematics and

Mathematical

Sciences
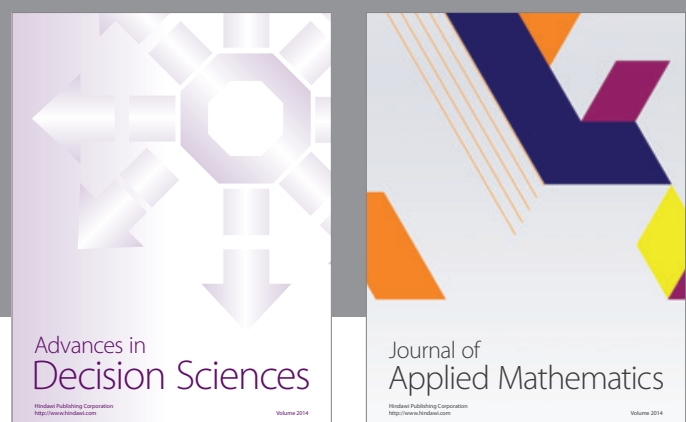

Journal of

Applied Mathematics
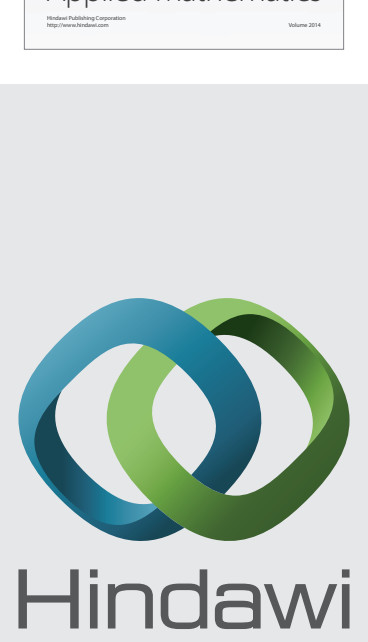

Submit your manuscripts at http://www.hindawi.com
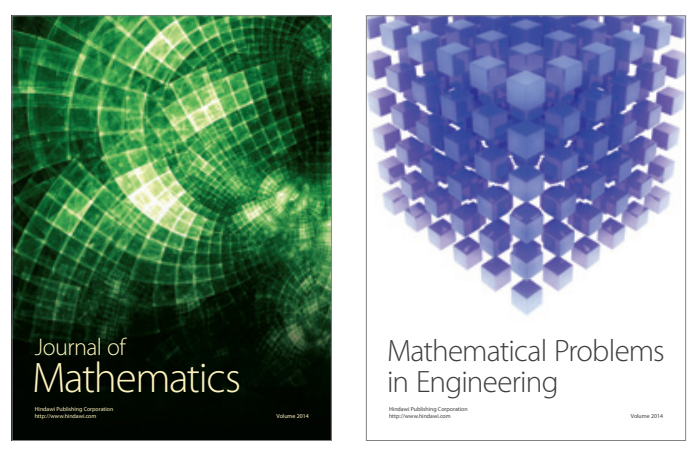

Mathematical Problems in Engineering
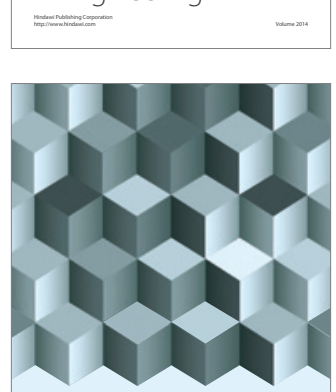

Journal of

Function Spaces
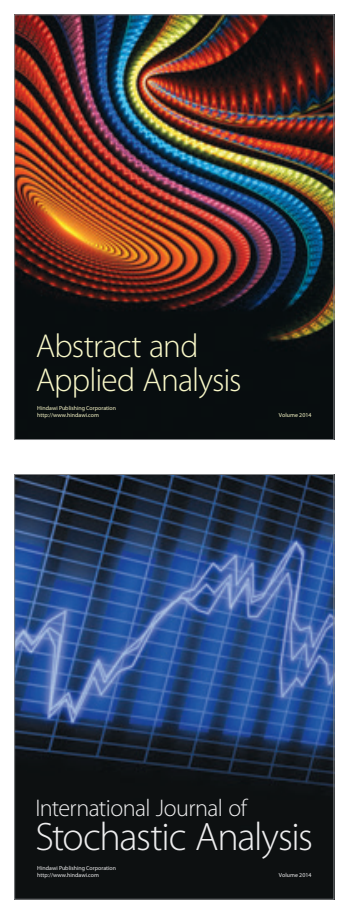

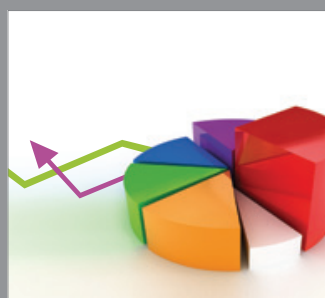

ournal of

Probability and Statistics

Promensencen
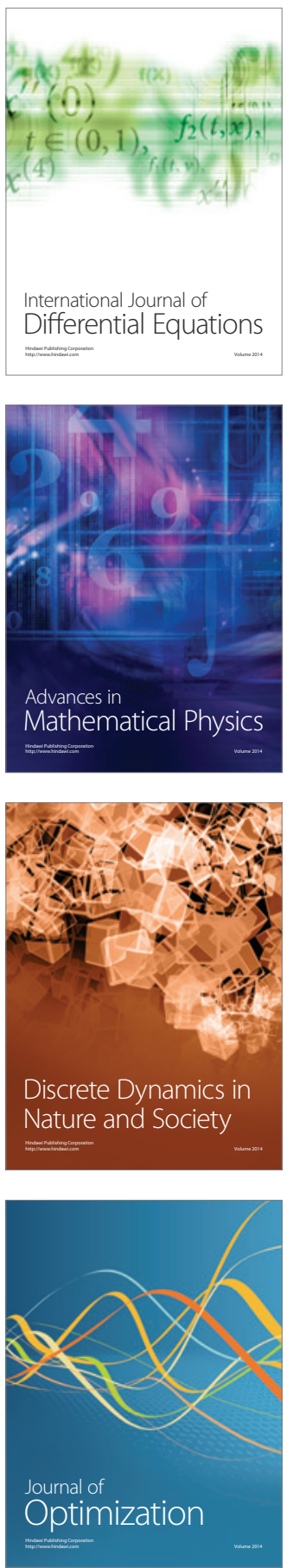